\title{
Jamming dynamics in grain mixtures : An extended hydrodynamic approach
}

\author{
Supurna Sinha \\ Raman Research Institute, Bangalore 560 080,India
}

(Dated: November 16, 2018)

\begin{abstract}
We study jamming in granular mixtures from the novel point of view of extended hydrodynamics. Using a hard sphere binary mixture model we predict that a few large grains are expected to get caged more effectively in a matrix of small grains compared to a few small grains in a matrix of larger ones. A similar effect has been experimentally seen in the context of colloidal mixtures.

PACS numbers: PACS numbers: 87.15.-v,05.40.-a,36.20.-r
\end{abstract}




\section{INTRODUCTION}

In recent years granular matter has emerged as an active area of research[1]. Interest in this field has grown as a result of observations coming from a large number of interesting and

relatively low-tech experiments [2]. In particular, a vibrated granular system [3] consisting of a large number of macroscopic grains in motion, provides us with a "scaled up" fluid system where we can explore the similarities and differences between such a large scale system and a microscopic atomic fluid.

Recent studies [1, 4, 5] in dense granular systems indicate that there is a striking similarity between the dynamics of granular materials and glassy dynamics in atomic fluids and colloids[6]. In this Letter, we exploit this analogy to understand some facets of jamming in granular matter. In particular, we study the role of compositional disorder in forming jammed configurations which slow down the dynamics and eventually result in a state of structural arrest characteristic of a glass [7, 8, 9, 10]. The observations made here are analogous to jamming effects studied in atomic liquid mixtures and colloids [7, 11, 12].

The behavior of a fluid at large length and time scales is well described by hydrodynamics. The hydrodynamic description has been extended to molecular length scales in an extended or generalized hydrodynamic description [7, 13]. In a generalized hydrodynamic framework the basic structure of the hydrodynamic equations is retained and the static susceptibilities and transport coefficients are wave-vector dependent to account for the nontrivial static correlations that come into play on molecular scales in a dense liquid. The generalized hydrodynamic description has proved to be very successful in describing the dramatic narrowing of the central diffusive peak in a neutron scattering spectrum $S(k, \omega)$ of a dense liquid[14]. Such a slow decay of density fluctuations is a precursor to glassy dynamics in dense liquids. A binary liquid mixture turns out to be more effective in forming glasses compared to a one component liquid since compositional disorder leads to jammed configurations which prevent the system from reaching its global equilibrium crystalline configuration[15]. Generalized hydrodynamic studies of dense binary hard sphere mixtures in the context of glass transition suggest that at intermediate wavevectors (i.e. equivalently on length scales of the order of the average of the molecular diameters of the two species) the density fluctuations of the two species emerge as the slowest decaying fluctuations and therefore dominate the slow dynamics of the system [7, 12]. In a self-consistent mode-coupling theory (MCT), nonlinear 
couplings of these slowly decaying modes of density fluctuations lead to a glassy state where structural relaxation is frozen [8, 9].

In this Letter we use, for the first time, generalized hydrodynamic techniques developed in the realm of atomic liquids to understand the behavior of jammed configurations in granular mixtures. Such an approach to granular matter enables us to take into consideration nontrivial static correlations stemming from the granularity or finite sizes of the particles, which in turn, influences the high density jamming dynamics of the system. We thus gain new insight into granular mixture dynamics and make predictions for future experiments in such systems.

\section{EXTENDED HYDRODYNAMIC APPROACH TO A BINARY MIXTURE OF GRAINS}

Consider a binary granular mixture of hard spheres of diameters $\sigma_{1}$ and $\sigma_{2}\left(\sigma_{2}>\sigma_{1}\right)$, masses $m_{1}$ and $m_{2}$, number densities $n_{1}$ and $n_{2}$ and of total packing fraction $\eta=$ $\frac{\pi}{6}\left[n_{1} \sigma_{1}^{3}+n_{2} \sigma_{2}^{3}\right]$. An analysis of the extended hydrodynamic equations of such a binary hard sphere mixture suggests that on length scales of the order of the size of the hard sphere particles [7] momentum and temperature fluctuations decay very fast and the slow dynamics of the system can be well described in terms of the modes of density fluctuations of the two species [12, 16]. This enables us to confine to a two mode description of the system on length scales of the order of the diameters of the hard sphere granular particles at high densities. It is convenient to describe the system in terms of the following linear combinations of the density fluctuations of the two species- the total mass density fluctuation at wavevector $\vec{k}$

$$
\rho_{\vec{k}}=\rho_{1 \vec{k}}+\rho_{2 \vec{k}}
$$

and the concentration fluctuation at wavevector $\vec{k}$

$$
c_{\vec{k}}=\frac{\rho_{2}}{\rho^{2}} \rho_{1 \vec{k}}-\frac{\rho_{1}}{\rho^{2}} \rho_{2 \vec{k}}
$$

where $\rho_{1 \vec{k}}$ and $\rho_{2 \vec{k}}$ are the mass density fluctuations of species 1 and $2, \rho_{1}=m_{1} n_{1}$ and $\rho_{2}=m_{2} n_{2}$ are the equilibrium mass densities of species 1 and 2 and $\rho=\rho_{1}+\rho_{2}$ is the total equilibrium mass density. The set of Laplace transformed coupled extended hydrodynamic 
equations of this system is given by:

$$
\left[z+\frac{k^{2}}{\rho \chi_{T}(k) \gamma_{L}(k)}\right] \rho_{\vec{k}}(z)+\frac{k^{2} \rho}{\beta \gamma_{L}(k)}\left[\frac{f_{1}(k)}{m_{1}}-\frac{f_{2}(k)}{m_{2}}\right] c_{\vec{k}}(z)=\rho_{\vec{k}}(t=0)
$$

and

$$
\begin{gathered}
{\left[z+k^{2} D(k)\left[\frac{m_{2}}{m_{1}} \sqrt{\frac{n_{2}}{n_{1}}} f_{1}(k)+\frac{m_{1}}{m_{2}} \sqrt{\frac{n_{1}}{n_{2}}} f_{2}(k)\right]\right] c_{\vec{k}}(z)} \\
+\frac{k^{2}}{\rho^{2}} D(k) \sqrt{n_{1} n_{2}}\left[m_{1} f_{2}(k)-m_{2} f_{1}(k)\right] \rho_{\vec{k}}(z)=c_{\vec{k}}(t=0)
\end{gathered}
$$

where $\chi_{T}(k)$ is the generalized isothermal compressibility, defined in terms of the partial static structure factors $S_{i j}(k)$ with $i=1,2$ and $j=1,2$ :

$$
\chi_{T}(k)=\chi_{T}^{0} \frac{S_{11}(k) S_{22}(k)-S_{12}^{2}(k)}{x_{2} S_{11}(k)+x_{1} S_{22}(k)-2 \sqrt{x_{1} x_{2}} S_{12}(k)}
$$

Here $\chi_{T}^{0}$ is the compressibility of the granular gas in the dilute limit. $\gamma_{L}(k)$ is the generalized longitudinal viscosity [7].

$$
f_{1}(k)=\frac{S_{22}(k)+\frac{m_{1}}{m_{2}} \sqrt{\frac{n_{1}}{n_{2}}} S_{12}(k)}{S_{11}(k) S_{22}(k)-S_{12}^{2}(k)}
$$

and

$$
f_{2}(k)=\frac{S_{11}(k)+\frac{m_{2}}{m_{1}} \sqrt{\frac{n_{2}}{n_{1}}} S_{12}(k)}{S_{11}(k) S_{22}(k)-S_{12}^{2}(k)}
$$

are combinations of partial static structure factors and $D(k)$ is the coefficient of mutual diffusion[7, 17].

This set of equations leads to two extended hydrodynamic diffusive modes.

Since we are interested in exploring the packing aspects of jamming in a binary granular mixture which is controlled by the sizes rather than the masses of the particles, we confine ourselves to the case of equal masses $m_{1}=m_{2}$ for the two species[15]. Here we analyze two illuminating special cases to bring out the role of size difference and packing in the jamming process which triggers the transition to a glassy state: $(i)$ a system composed of a few large spheres in a matrix of small spheres and $(i i)$ a system composed of a few small spheres in a matrix of large spheres.

In both these extreme limits $\left(x_{2}=n_{2} / n<<1\right.$ and $\left.x_{1}=n_{1} / n<<1\right)$ the cross terms in the expressions representing the eigenvalues for the extended diffusive modes are negligibly small and the mode structure reduces to:

$$
z_{-}(k) \simeq-k^{2} \frac{1}{\rho \chi_{T}(k) \gamma_{L}(k)}
$$


and

$$
z_{+}(k) \simeq-k^{2} D(k)\left[\sqrt{\frac{n_{2}}{n_{1}}} f_{1}(k)+\sqrt{\frac{n_{1}}{n_{2}}} f_{2}(k)\right]
$$

Thus, there are two relevant modes: $z_{-}(k)$, which governs the relaxation of total mass density fluctuations and $z_{+}(k)$, which governs the relaxation of concentration fluctuations. Let us analyze these modes for case (i). In this case, since the mixture consists of a large number of small spheres, the static compressibility $\chi_{T}(k)$ which is the main determinant of the dynamics of a dense liquid, is given by $\chi_{T}(k) \simeq \chi_{T}^{0} S_{11}(k)$, i.e. it is dominated by the static structure factor of the small spheres. Thus, $z_{-}(k) \simeq-k^{2} \frac{1}{\rho \chi_{T}^{0} S_{11}(k) \gamma_{L}(k)}$. Consequently there is a significant slowing down of the dynamics of density fluctuations of the background matrix at the location of the first peak of $S_{11}(k)[18]$. In this case the static structure of large (type 2 ) spheres is flat and is given by $S_{22}(k) \simeq 1$. Thus, $z_{+}(k) \simeq-k^{2} \frac{D_{02}}{S_{22}(k)} \simeq-k^{2} D_{02}$, where $D_{02}$ is the dilute gas limit of the diffusion coefficient of large spheres[17]. In case (ii), the large (type 2) and small (type 1) spheres switch roles. The final picture that emerges is the following. Caging and slowing down of dynamics is more effective for $x_{2}<<1$ firstly because the ratio $D_{02} / D_{01}=\sigma_{1} / \sigma_{2}<1$ [7, 19] and a few large spheres diffuse slower in the background of small spheres compared to a few small spheres in the background of large spheres. In addition, in both cases there is also a significant slowing down of structural relaxation due to softening of the mode of total density fluctuations $z_{-}(k)$ around the location of the peak of the static structure factor of the majority particles.

In other words, caging is more efficient in a mixture dominated by small spheres compared to one dominated by large spheres. This is the main prediction made in this Letter for future experiments on dense granular mixtures designed to probe the efficiency of caging in such systems.

The main point that we emphasize in this Letter is that by exploiting the analogy between glass forming atomic liquids and granular matter we can draw some definite testable conclusions about caging dynamics in grains.

To summarize, we have, for the first time applied extended or generalized hydrodynamic techniques to dynamics of granular matter. In particular, we consider a dense binary mixture of hard spheres and analyze the modes of density fluctuations which dominate the slow dynamics on length scales of the order of the sizes of the granular particles constituting the system. Our analysis points to some differences in jamming behavior between a mixture 
dominated by small spheres and one dominated by large spheres. Effects similar to the ones predicted here have been observed in confocal microscopy studies in colloidal mixtures [11]. The predictions made here can be tested against experiments in vibrated dense granular mixtures. We expect our analysis to be valid for a granular mixture consisting of nearly elastic spheres of comparable masses [20]. While the present analysis captures the onset of glassy behavior in granular mixtures, it would be worthwhile to do a mode-coupling study for such a system using the extended hydrodynamic modes that stem out of our analysis as an input to understand glass transition in granular mixtures. In future, one can do a more complete analysis where the effects of momentum and temperature fluctuations and inelastic collisions [21] are taken into consideration and check if the high density particlescale dynamics presented here survives the inclusion of these effects.

[1] See for instance, H. A. Maske, J. Brujic and S. F. Edwards, The physics of Granular Media, Wiley-VCH, (2004).

[2] See for instance, K. Feitosa and N. Menon, Physical Review Letters 88 , 198301 (2002).

[3] X. Yang, C. Huan, D. Candela, R. W. Mair and R. L. Walsworth Phys. Rev. Lett. 88, 044301 (2002); X. Yang, C. Huan, D. Candela, R. W. Mair and R. L. Walsworth Physical Review E 69, 041302 (2004);

[4] A. J. Liu and S. R. Nagel, Nature 396, 21 (1998).

[5] See for instance M. Nicodemi et al, "Statistical Mechanics of jamming and segregation in granular media" in "Unifying Concepts in Granular Media and Glasses" edts. A. Coniglio, A. Fierro, H. J. Herrmann and M. Nicodemi.

[6] W. Gotze and L. Sjorgen, Rep. Prog. Phys 55, 241 (1992).

[7] M. C. Marchetti and Supurna Sinha, Physical Review A 413214 (1990); Supurna Sinha and M. C. Marchetti, Physical Review A 464942 (1992).

[8] U. Harbola and S. P. Das Physical Review E 65, 036138 (2002).

[9] J. Bosse and J. S. Thakur, Physical Review Letters 59, 998 (1987).

[10] J. Bosse and Y. Kaneko, Physical Review Letters 74, 4023 (1995).

[11] A. D. Dinsmore, E. R. Weeks, V. Prasad, A. Levitt and D. A. Weitz, Applied Optics 40, 4152 (2001). 
[12] Supurna Sinha Physical Review E 493504 (1994).

[13] T. R. Kirkpatrick, Physical Review A 32, 3130 (1985).

[14] P. G. de Gennes, Physica 25, 825 (1959).

[15] J. N. Roux, J. L. Barrat and J. P. Hansen Journal Of Physics : Condensed Matter 1, 7171 (1989).

[16] A more familiar example is the phenomenon of de Gennes narrowing which happens in the context of dense simple liquids.

[17] J. Ferziger and H. Kaper, Mathematical Theory Of Transport Processes in Gases, NorthHolland, Amsterdam, (1972).

[18] Also, we are focussing on a high viscosity (i.e. large $\left.\gamma_{L}(k)[[7,13]]\right)$ regime which contributes to the process of structural slowing down.

[19] See for instance, G. V. Vijayadamodar and B. Bagchi Journal Of Chemical Physics 93, 689 (1990).

[20] In such a domain the mixture can be described fairly well as one sharing a common temperature T. See for instance P. Zamankhan Physical Review E 52, 4877 (1995) and V. Garzo and J. W. Dufty Physical Review E 60, 5706 (1999).

[21] See, for instance, J. W. Dufty and J. J. Brey Physical Review E 68, 030302 (2003); J. J. Brey and J. W. Dufty Physical Review E 72, 011303 (2005); 